\title{
Three-dimensional comparison of the skeletal and dentoalveolar effects of the Herbst and Pendulum appliances followed by fixed appliances: A CBCT study
}

\author{
Kyle L. Taylor ${ }^{1}$ (D) | Karine Evangelista ${ }^{1,2}$ (D) | Luciana Muniz ${ }^{1}$ (D) | \\ Antônio Carlos de Oliveira Ruellas ${ }^{1}$ (D) | José Valladares-Neto ${ }^{2}$ (i) | James McNamara Jr ${ }^{1}$ | \\ Lorenzo Franchi $^{3}$ (D) | Hera Kim-Berman ${ }^{1}$ (D) | Lucia Helena Soares Cevidanes ${ }^{1}$ (D)
}

\author{
${ }^{1}$ Department of Orthodontics and Pediatric \\ Dentistry, School of Dentistry, University of \\ Michigan, Ann Arbor, MI, USA \\ ${ }^{2}$ School of Dentistry, Federal University of \\ Goiás, Goiânia, Brazil \\ ${ }^{3}$ Department of Experimental and Clinical \\ Medicine, Orthodontics, Università degli \\ Studi di Firenze, Florence, Italy

\section{Correspondence} \\ Karine Evangelista, School of Dentistry, \\ Federal University of Goiás, Avenida \\ Universitária esquina com $1^{\text {a }}$ Avenida, s/ \\ número. 74605-220, Goiânia, State of Goiás, \\ Brazil. \\ Email: kemar_7@hotmail.com \\ Funding information \\ Coordenação de Aperfeiçoamento de \\ Pessoal de Nível Superior, Grant/Award \\ Number: 001; National Institute of Dental \\ and Craniofacial Research, Grant/Award \\ Number: R01 DE024450
}

\author{
Abstract \\ Objectives: To compare three-dimensional (3D) skeletal and dentoalveolar effects \\ of the Herbst and Pendulum appliances followed by fixed orthodontic treatment in \\ growing patients.
}

Setting and sample population: A sample of 35 adolescents with cone-beam computed tomography scans obtained prior to Herbst and Pendulum treatment (T1) and immediately after fixed appliance treatment (T2).

Materials and Methods: Patients with Class II malocclusion was assessed retrospectively and divided into two treatment groups: Herbst group ( $\mathrm{n}=17$, age: $12.0 \pm 1.6$ years) and Pendulum group ( $n=18$, age: $12.1 \pm 1.5$ years), with a mean treatment duration of $2.8 \pm 0.8$ years and $2.5 \pm 0.7$ years, respectively. Reconstructions of the maxillomandibular and dentoalveolar regions and data in 3D were obtained relative to cranial base, maxillary and mandibular regional superimpositions. Treatment outcomes (T2-T1) were compared between both groups using $t$ tests for independent samples $(P<.05)$.

Results: Significant increase in mandibular length was observed in the Herbst group $(7.3 \pm 3.5 \mathrm{~mm})$ relative to the Pendulum group $(4.6 \pm 4.5 \mathrm{~mm})$. Inferior and anterior displacements of Pogonion were $2.2 \mathrm{~mm}$ and $1.6 \mathrm{~mm}$ greater in the Herbst group, respectively. The mesial displacement of the lower first molars was significantly greater in the Herbst group (1.9 mm). The upper first molars had contrasting results in sagittal displacement, with $0.6 \pm 1.7 \mathrm{~mm}$ of distal displacement with the Pendulum and $1.4 \pm 2.1 \mathrm{~mm}$ of mesial displacement with the Herbst. Lower incisor projection and proclination were similar between groups.

Conclusions: The Herbst and Pendulum appliances followed by comprehensive orthodontic treatment effectively corrected Class II malocclusion in growing patients, but with differing skeletal and dentoalveolar effects.

KEYWORDS

Angle Class II malocclusion, cone-beam computed tomography, Herbst appliance, Pendulum appliance, three-dimensional images 


\section{1 | INTRODUCTION}

Over the years, both fixed and removable intraoral appliances have become increasingly popular for the correction of Class II malocclusion, with the Herbst appliance being one of the most widely used worldwide. Unlike removable appliances and elastics, the Herbst appliance is fixed to the teeth and reduces the need for active patient compliance. ${ }^{1}$ The Pendulum appliance is another alternative to treat Class II malocclusion that does not require patient cooperation. ${ }^{2,3}$ However, both devices presumably handle Class II treatment differently. The Herbst appliance produces a forward repositioning of the lower jaw as the patient closes into occlusion; increased growth rates and decreased facial convexity have been reported. ${ }^{1}$ The Herbst appliance has also been shown to distalize and intrude the maxillary first molars. ${ }^{4,5}$ The Pendulum appliance is designed primarily for maxillary molar distalization in non-extraction cases. ${ }^{2}$

Due to their presumably different paths to treat Class II malocclusion, it is expected that Pendulum and Herbst appliances may produce different specific skeletal and dentoalveolar changes. Previous 2D studies of the Pendulum appliance have noted clockwise mandibular rotation secondary to distalization of the maxillary molars. ${ }^{2}$ Moreover, a significant restriction of anterior maxillary displacement has been observed. ${ }^{6}$ Molar distalization accounted for $63 \%-76 \%$ of the space opening, and $24 \%-37 \%$ was due to maxillary second premolar mesialization. ${ }^{2,7}$

Herbst treatment studies have reported restriction of maxillary growth, mandibular rotation changes, as well as anterior positioning of the glenoid fossa ${ }^{8-10}$ and a favourable impact on the advancement of the chin. ${ }^{8-11}$ Recently, a 3D study conducted with cone-beam computed tomography (CBCT) images has demonstrated that patients with different vertical facial patterns and Class II malocclusion show similar patterns of mandibular and maxillary changes when treated with the Herbst appliance relative to the magnitude and direction of condylar growth, ${ }^{12}$ as compared to fixed appliances and Class II intermaxillary elastics. ${ }^{13}$

Clinical questions remain regarding whether patients treated with the Herbst and Pendulum appliances present greater skeletal or dentoalveolar effects and following comprehensive treatment with fixed appliances. ${ }^{4,5,14}$ Three-dimensional (3D) assessment of the skeletal and dentofacial changes with Pendulum treatment compared to Herbst treatment followed by comprehensive fixed appliances may further the clinician's understanding of their treatment choice for each patient. Thus, the aims of this study were to use 3D imaging and superimposition techniques to assess skeletal and dentoalveolar changes associated with Class II correction in growing children treated either with the Herbst or Pendulum appliance followed by comprehensive treatment using fixed appliances.

\section{2 | SUBJECTS AND METHODS}

The Health Sciences and Behavioral Sciences Institutional Review Board at University of Michigan determined that this observational retrospective study was exempt from IRB oversight (HUM00123010).

The sample was selected from a single orthodontic office, using information following inclusion criteria: (a) pre-treatment Class II Division 1 malocclusion defined by at least an end-to-end molar relationship; (b) cervical vertebral maturation (CVM) stage of CS2-4 ${ }^{15}$; (c) treatment with Herbst or Pendulum therapy (Phase 1) immediately followed by pre-adjusted edgewise appliance treatment (Phase 2), where both treatment options were presented to the patients and parents based on the orthodontist evaluation of mandibular retrusion, (d) no permanent teeth extracted before or during treatment; (e) CBCT images of good quality and with adequate field of view (FOV) taken before treatment (T1) and immediately after removal of the pre-adjusted edgewise appliances (T2); and (f) post-treatment images showing Class I relationship of canines and molars. Exclusion criteria included (a) T1 to T2 interval was >48 months; (b) T1 to T2 interval was $<12$ months; (c) history of previous orthodontic treatment; and (d) use of temporary anchorage devices during treatment.

Table 1 shows the starting forms for sex, CVM stage, age, skeletal and dentoalveolar variables at baseline (T1) from both Herbst and Pendulum groups.

The sample size calculation (a minimum of 17 patients in each group) was based on a power of $80 \%$, an alpha of 0.05 , a difference of $2.0 \mathrm{~mm}$ for the anterior displacement of Pogonion (Pog to nasion perpendicular) and a standard deviation for this variable of $2.0 \mathrm{~mm}$ (pooled standard deviation between Pendulum and stainless-steel crown Herbst groups was derived from a previous study). ${ }^{14}$

\section{1 | Herbst protocol}

The Herbst group ( $n=17$ ) had the same Herbst appliance design with bilateral telescopic tubes and plungers attached to a maxillary and mandibular metal framework. This rigid framework was soldered to thick stainless-steel crowns cemented on the maxillary first molars and the mandibular first premolars; occlusal rests also were bonded to the lower first molars. The fixed design of the Herbst ensured full-time wear of the appliance.

The Herbst treatment protocol for all patients consisted of (a) Herbst appliance installation and adjustment of the upper and lower incisors close to an edge-to-edge ( $0 \mathrm{~mm}$ overjet) relationship at the start of treatment; (b) evaluation for adequate mandibular advancement by the treating orthodontist every 3 months until Herbst removal; (c) Herbst appliance was maintained for 12 months; and (d) removal of the Herbst appliance and bonding of fixed appliances for comprehensive treatment.

\section{2 | Pendulum protocol}

The Pendulum group $(n=18)$ received a Pendulum appliance as described by Hilgers ${ }^{3}$ that consisted of bilateral distalizing springs anchored to the dorsal portion of the palatal acrylic button, made of 0.032 " titanium-molybdenum alloy (TMA) wire. The treatment protocol consisted of the following: (a) the distalizing springs 


\begin{tabular}{|c|c|c|c|c|c|c|c|}
\hline & \multicolumn{2}{|c|}{ Herbst $(n=17)$} & \multicolumn{2}{|c|}{$\begin{array}{l}\text { Pendulum } \\
(n=18)\end{array}$} & \multirow{2}{*}{$\begin{array}{l}\text { Mean } \\
\text { Diff }\end{array}$} & \multirow{2}{*}{$\begin{array}{l}95 \% \mathrm{Cl} \\
\text { of mean } \\
\text { diff }\end{array}$} & \multirow[b]{2}{*}{$P$ value } \\
\hline & Mean & SD & Mean & SD & & & \\
\hline $\begin{array}{l}{ }^{\mathrm{a} S e x} \text { (female/ } \\
\text { male) \% }\end{array}$ & \multicolumn{2}{|l|}{$52.9 / 47.1$} & \multicolumn{2}{|c|}{$83.3 / 11.8$} & & & .029 \\
\hline $\begin{array}{l}{ }^{\mathrm{b}} \text { CVM stages } \\
(2 / 3 / 4) \%\end{array}$ & \multicolumn{2}{|c|}{$29.4 / 35.3 / 35.3$} & \multicolumn{2}{|c|}{$11.1 / 38.9 / 50.0$} & & & .410 \\
\hline Age $(y)$ & 12.0 & 1.6 & 12.1 & 1.5 & -0.03 & $-1.1 ; 1.0$ & .947 \\
\hline $\begin{array}{l}\text { Treatment dura- } \\
\text { tion (y) }\end{array}$ & 2.8 & 0.8 & 2.5 & 0.7 & 0.4 & $-0.2 ; 0.9$ & .165 \\
\hline \multicolumn{8}{|l|}{ Vertical skeletal } \\
\hline SN.GoGn $\left(^{\circ}\right)$ & 30.3 & 5.7 & 29.7 & 4.2 & 0.8 & $-2.6 ; 4.2$ & .636 \\
\hline \multicolumn{8}{|l|}{ Maxillary skeletal } \\
\hline Co-A (mm) & 82.4 & 5.9 & 79.1 & 3.9 & 3.2 & $-0.2 ; 6.6$ & .064 \\
\hline $\begin{array}{l}\text { Maxillary } \\
\text { length (ANS- } \\
\text { PNS-mm) }\end{array}$ & 50.1 & 3.8 & 49.6 & 3.1 & 0.6 & $-1.7 ; 3.0$ & .660 \\
\hline \multicolumn{8}{|c|}{ Mandibular skeletal } \\
\hline $\begin{array}{l}\text { Mandible } \\
\text { length } \\
\text { (CoGn-mm) }\end{array}$ & 102.4 & 5.2 & 100.4 & 6.1 & 1.7 & $-2.2 ; 5.6$ & .379 \\
\hline Co.GoMe $\left(^{\circ}\right)$ & 123.3 & 5.7 & 119.8 & 4.8 & 3.6 & $-0.1 ; 7.2$ & .052 \\
\hline \multicolumn{8}{|c|}{ Maxillary/Mandibular } \\
\hline $\operatorname{ANB}\left({ }^{\circ}\right)$ & 5.0 & 1.7 & 4.0 & 1.7 & 1.0 & $-0.2 ; 2.2$ & .095 \\
\hline \multicolumn{8}{|c|}{ Maxillary dentoalveolar } \\
\hline $\mathrm{U} 1$ to $\mathrm{SN}\left({ }^{\circ}\right)$ & 105.90 & 11.4 & 102.4 & 7.4 & 3.2 & $-3.4 ; 9.7$ & .330 \\
\hline \multicolumn{8}{|c|}{ Mandibular dentoalveolar } \\
\hline IMPA & 92.9 & 7.3 & 90.8 & 4.4 & 1.8 & $-2.4 ; 6.0$ & .385 \\
\hline
\end{tabular}

TABLE 1 Statistical comparison for sex, cervical vertebral maturation (CVM) stage, age, and skeletal and dentoalveolar variables at baseline (T1) and treatment duration (T1-T2)

${ }^{\text {a}}$ Fischer's exact test.

${ }^{\mathrm{b}} \mathrm{Qui}$-square

$t$ tests $(P<.05)$.

were placed in their pre-activate state into palatal sheaths of the molar bands; (b) initial activation of $60^{\circ}$ to $70^{\circ}$ (about the width of one molar) was incorporated to generate approximately $250 \mathrm{~g}$ of force per side; (c) patients were monitored every 4 to 6 weeks until adequate distalization with achievement of Class I molar relationship was determined by the treating orthodontist; and (d) after distalization, the appliance was removed, and a Nance button banded to the molars was placed along with fixed orthodontic appliances.

All patients in both groups had a subsequent phase of fixed preadjusted edgewise appliances immediately following the respective Phase I treatment. Class II elastics were worn during the phase of fixed pre-adjusted edgewise appliances as needed to achieve final canine Class I intercuspation.

\section{3 | Image analysis}

CBCT scans had been taken for diagnostic and clinical purposes for all subjects in T1 and T2, using an iCat unit (Imaging Sciences International), with 40 seconds scan time, as well as a $23 \times 17-\mathrm{cm}$
FOV and a voxel size of $0.4 \mathrm{~mm}^{3}$, following the ALARA principle. All images were exported as DICOM files.

An orthodontist examiner, previously trained in using the following method, performed all image analysis procedures. 3D surface models were created following 10 image analysis steps:

1. DICOM files were converted to GIPL files in order to decrease the file size using ITK-SNAP, an open source software (version 2.4.0; www.itksnap. org);

2. The $0.4 \mathrm{~mm}^{3}$ original voxel size was converted to $0.5 \mathrm{~mm}^{3}$ using 3D Slicer (version 4.0; www.slicer.org) to decrease the computational power and time for cranial base registration;

3. Volumetric label maps of the entire cranial complex at T1 and T2 were created using ITK-SNAP;

4. $3 D$ surface models at $T 1$ were created using $3 D$ Slicer;

5. Head orientation of T1 model using the 3D standardized coordinate system of the 3D Slicer software as reference, setting the Frankfort plane (bilateral Orbitale and Porion) perpendicular to the midsagittal plane (Glabella, Crista Galli and Basion), as described by Ruellas et al $^{16}$ 


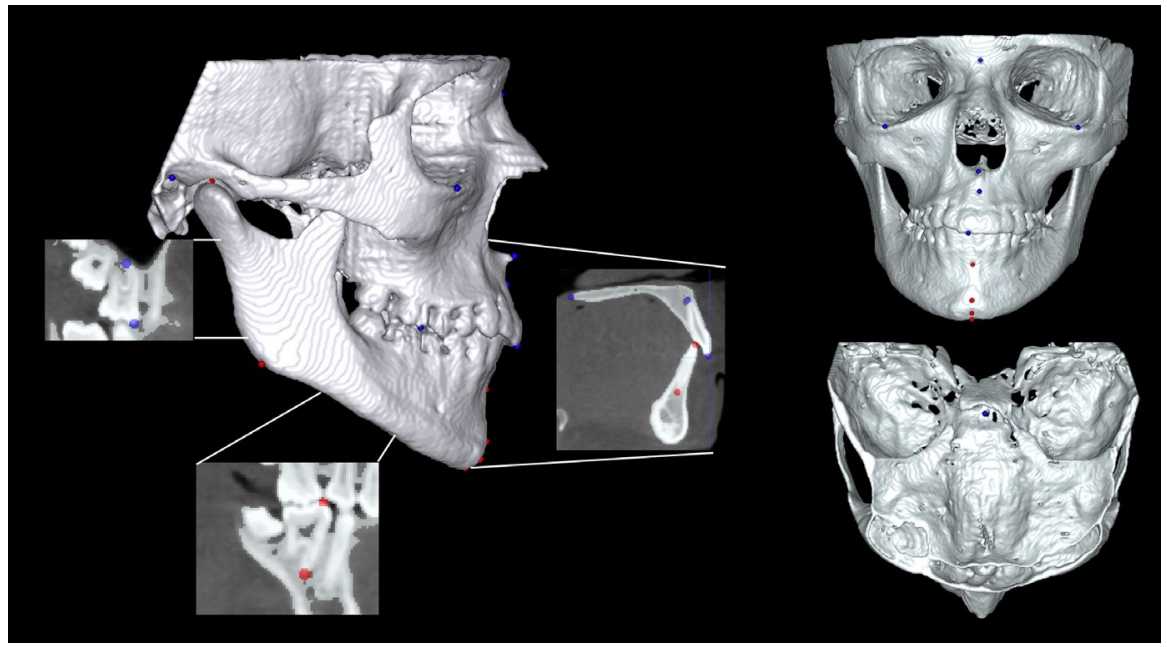

FIGURE 1 3D Segmented model with labelled landmarks. Blue landmarks: sella (S)-midpoint of the pituitary fossa (sella turcica) in axial and sagittal views; nasion (N)-most anterior and middle point of the frontonasal suture in axial and sagittal views; orbitale (Or)-lowest point in the inferior margin of the orbit in axial and sagittal views; porion (Po)-superior point of the external auditory meatus in axial and sagittal views; anterior nasal spine (ANS)-tip of the median, sharp bony process of the maxilla at the lower margin of the anterior nasal in coronal and sagittal views; posterior nasal spine (PNS)-the most posterior point at the sagittal plane on the bony hard palate in coronal and sagittal views; point $\mathrm{A}(\mathrm{A})$-the most posterior point on the curve of the maxilla between the anterior nasal spine and supradentale in coronal and sagittal views; upper incisor tip (U1T)-the incisal tip of the upper central incisor in axial and sagittal views; upper incisor apex (U1A)-the root tip of the upper central incisor in axial and sagittal views; upper molar tip (U6T)-the buccal mesial cusp tip of the maxillary first molar in axial and sagittal views; and upper molar apex (U6A)-the apex of the mesial root of the maxillary first molar in axial and sagittal views. Red landmarks: condylion (Co)the most posterosuperior point on the curvature of the average of the right and left outlines of the condylar head in axial and sagittal views; gonion (Go)-the midpoint of the angle of the mandible in axial and sagittal views; menton (Me)-most inferior midline point on the mandibular symphysis in axial and sagittal views; gnathion (Gn)-the most anteroinferior point on the contour of the bony chin symphysis in axial and sagittal views; pogonion (PG)-the most anterior point on the contour of the bony chin in axial and sagittal views; lower incisor tip (L1T)-the incisal tip of the mandibular central incisor in axial and sagittal views; lower incisor apex (L1A)-the root tip of the mandibular central incisor in axial and sagittal views; lower molar cusp tip (L6T)-the anterior cusp tip of the mandibular first molar in axial and sagittal views; lower molar apex (L6A)the apex of the mesial root of the mandibular first molar in axial and sagittal views [Colour figure can be viewed at wileyonlinelibrary.com]

6. The T2 scan was approximated to the oriented T1 scan using three different regions as reference, one at time;

a. Cranial base: using the anterior cranial fossa as a best fit reference in order to evaluate the skeletal positional or growth changes in maxilla and mandible ${ }^{17}$;

b. Maxilla: using the maxillary palatal plane and anterior nasal spine as a best fit reference to evaluate the upper dentoalveolar changes ${ }^{18}$

c. Mandible: using the mandible base and symphysis as a best fit reference in order to evaluate the lower dentoalveolar changes ${ }^{19}$;

7. Fully automated voxel-based registrations of the cranial base, maxilla and mandible were performed in 3D Slicer using the respective scans and segmentation of T2 approximated;

8. Pre-labelling: landmarks (Figure 1 ) were pre-labeled ${ }^{18}$ using the multiplanar views, axial, coronal and sagittal, at T1 and T2 using ITK-snap, and were positioned using at least two sectional views simultaneously for orientation.

9. Virtual 3-dimensional (3D) surface models with landmarks were created for T1 and T2 using 3D slicer

The linear distances and angles, as well as the 3D changes (Table 2), were determined, and the amount of directional changes in each plane of 3D space was measured between corresponding coordinates of landmarks placed in the T1 and T2 surface models using 3D Slicer Q3DC (Quantification of 3D Components) tool in the 3D Slicer. The skeletal and dentoalveolar variables are also described in Table 2.

\subsection{Statistical Analysis}

To determine intra-rater reliability, 10 patients were selected randomly from both groups and tested using intraclass correlation coefficients (ICC) test with $95 \% \mathrm{Cl}$. The measurements were repeated 2 weeks later. Data normality was tested by the Shapiro-Wilk test. Chi-square and Fisher's exact tests compared, respectively, CVM stage and sex, and $t$ tests for independent samples were used to compare all other variables. All data were analysed with a social science statistical package (SPSS, version 25.0, SPSS, Inc), and the significance was tested at $P<.05$.

\section{3 | RESULTS}

The ICC test resulted in excellent repeatability scores for all skeletal and dentoalveolar measurements (Table 3). Descriptive statistics including means and standard deviations for each group at the start of 


\begin{tabular}{|c|c|c|c|}
\hline Craniofacial relationships & Measures & Abbreviation & Direction \\
\hline \multirow[t]{4}{*}{ Maxillary skeletal } & Point A displacement & $\mathrm{A}(\mathrm{mm})$ & $\mathrm{AP}, \mathrm{SI}, 3 \mathrm{D}$ \\
\hline & Point ANS displacement & ANS (mm) & $\mathrm{AP}, \mathrm{SI}, 3 \mathrm{D}$ \\
\hline & Maxillary length change & ANS-PNS (mm) & $3 D$ \\
\hline & Maxillary plane change & ANS-PNS $\left({ }^{\circ}\right)$ & Pitch \\
\hline \multirow[t]{4}{*}{ Maxillary dentoalveolar } & $\begin{array}{l}\text { Central incisor displace- } \\
\text { ment (U1T) }\end{array}$ & U1 (mm) & AP, SI, 3D \\
\hline & $\begin{array}{l}\text { Maxillary first molar } \\
\text { displacement (U6T) }\end{array}$ & $\mathrm{U} 6(\mathrm{~mm})$ & $\mathrm{AP}, \mathrm{SI}, 3 \mathrm{D}$ \\
\hline & $\begin{array}{l}\text { Incisor inclination } \\
\text { change (U1T-U1A) }\end{array}$ & U1 Inclination $\left({ }^{\circ}\right)$ & Pitch \\
\hline & $\begin{array}{l}\text { Molar angulation } \\
\text { change (U6T-U6A) }\end{array}$ & U6 Angulation $\left(^{\circ}\right)$ & Pitch \\
\hline \multirow[t]{3}{*}{ Mandibular Skeletal } & $\begin{array}{l}\text { Point Pogonion } \\
\text { displacement }\end{array}$ & Pog (mm) & $A P, S I, 3 D$ \\
\hline & $\begin{array}{l}\text { Mandibular plane } \\
\text { change }\end{array}$ & GoMe $\left(^{\circ}\right)$ & Pitch \\
\hline & Mandibular length & CoGn (mm) & $3 \mathrm{D}$ \\
\hline \multirow[t]{4}{*}{ Mandibular Dentoalveolar } & $\begin{array}{l}\text { Central incisor displace- } \\
\text { ment (L1T) }\end{array}$ & $\mathrm{L} 1$ (mm) & $\mathrm{AP}, \mathrm{SI}, 3 \mathrm{D}$ \\
\hline & $\begin{array}{l}\text { Maxillary first molar } \\
\text { displacement (L6T) }\end{array}$ & L6 (mm) & $\mathrm{AP}, \mathrm{SI}, 3 \mathrm{D}$ \\
\hline & $\begin{array}{l}\text { Incisor inclination } \\
\text { change L1T-L1A }\end{array}$ & L1 Inclination $\left(^{\circ}\right)$ & Pitch \\
\hline & $\begin{array}{l}\text { Molar angulation } \\
\text { change L6T-L6A }\end{array}$ & L6 Angulation $\left({ }^{\circ}\right)$ & Pitch \\
\hline
\end{tabular}

TAB LE 2 3D linear distances and angles used in this study

Abbreviations: 3D, three-dimensional; AP, anteroposterior; SI, superoinferior.

treatment are presented in Table 1. The direction of all measurements was described using the 3D-slicer software. Positive values represented anterior, superior, counterclockwise, mesial angulation and buccal inclination displacement changes. Negative values showed posterior, inferior, clockwise, distal angulation and lingual inclination changes. The analysis of starting forms revealed that the two groups were statistically similar in cephalometric measurements, CVM stage, chronological age and treatment duration. The only exception was gender, where female patients were more prevalent in the Pendulum group (Table 1).

\section{1 | Skeletal changes}

Table 3 shows the comparison of maxillary and mandibular skeletal changes (T2-T1) relative to cranial base and regional superimpositions in both treatment groups. Maxillomandibular skeletal changes showed an overall forward and downward displacement in both groups.

Maxillary changes differed significantly between the two groups. The Herbst group presented greater anterior (mean difference at Point ANS by $1.4 \mathrm{~mm}$ ), inferior (mean difference at Point A by $1.8 \mathrm{~mm}$ ) and 3D (mean difference at Point ANS by $2.0 \mathrm{~mm}$ and Point A by $2.0 \mathrm{~mm}$ ) displacements, as well as greater changes in maxillary length (ANS-PNS $1.4 \mathrm{~mm}$ mean group difference). The palatal plane angle showed minor changes in the Herbst $\left(-0.6 \pm 2.2^{\circ}\right)$ and Pendulum $\left(-0.1 \pm 1.7^{\circ}\right)$ groups, with no differences between groups.

The Herbst group presented significantly greater mandibular skeletal changes $(7.3 \pm 3.5 \mathrm{~mm})$ than the Pendulum group $(4.6 \pm 4.5 \mathrm{~mm})$, with a mean difference of $2.9 \mathrm{~mm}$. The Herbst group also demonstrated a larger downward displacement at Pogonion of $2.2 \mathrm{~mm}$, a vector displacement at Pogonion of $3.1 \mathrm{~mm}$ and greater mandibular length (CoGn of $2.9 \mathrm{~mm}$ ). The anteroposterior changes also revealed greater anterior displacement of Pogonion in the Herbst group $(3.3 \pm 2.9 \mathrm{~mm})$ that was not significantly different when compared to the Pendulum group (1.8 $\pm 2.0 \mathrm{~mm}$; Table 3 ). Similarly, relative to the palatal plane, the mandibular plane angle showed small changes in the Herbst $\left(-0.6 \pm 2.2^{\circ}\right)$ and Pendulum $\left(-0.1 \pm 1.7^{\circ}\right)$ groups, with no statistically difference between them (Table 3 ).

\section{2 | Dentoalveolar changes}

Maxillary and mandibular dentoalveolar changes in both groups are shown in Table 3. The Herbst and Pendulum groups showed small changes in the anteroposterior displacement of the upper incisors (0.1 $\pm 2.6 \mathrm{~mm}$ and $0.5 \pm 2.7 \mathrm{~mm}$, respectively). The upper incisor inclination was similar in the Pendulum $\left(0.8 \pm 9.7^{\circ}\right)$ and Herbst groups $\left(0.3 \pm 8.7^{\circ}\right)$. The between-group differences in the incisor sagittal position and inclination were not statistically significant. 
TAB LE 3 Intraclass coefficient correlation of intra-rater reliability and comparison of maxillary and mandibular skeletal and dentoalveolar changes T1-T2 relative to cranial base and regional superimpositions

\begin{tabular}{|c|c|c|c|c|c|c|c|c|c|}
\hline & \multicolumn{2}{|l|}{ ICC } & \multirow{2}{*}{$\begin{array}{l}\text { Herbst } \\
(n=17) \\
\text { Mean }\end{array}$} & \multirow[b]{2}{*}{ SD } & \multicolumn{2}{|c|}{ Pendulum $(n=18)$} & \multirow{2}{*}{$\begin{array}{l}\text { Mean } \\
\text { Diff }\end{array}$} & \multirow{2}{*}{$\begin{array}{l}95 \% \mathrm{Cl} \text { of } \\
\text { mean diff }\end{array}$} & \multirow[b]{2}{*}{$P$ value } \\
\hline & Coeff. & $95 \% \mathrm{Cl}$ & & & Mean & SD & & & \\
\hline \multicolumn{10}{|l|}{ Maxillary skeletal changes } \\
\hline Point A AP (mm) & 0.92 & $0.84 ; 0.96$ & 1.9 & 1.8 & 1.1 & 1.2 & 0.7 & $-0.3 ; 1.8$ & .167 \\
\hline Point A SI (mm) & 0.98 & $0.97 ; 0.99$ & -2.7 & 2.7 & -0.9 & 1.1 & -1.8 & $-0.4 ;-0.3$ & $.020^{*}$ \\
\hline Point ANS AP (mm) & 0.98 & $0.92 ; 0,99$ & 2.6 & 2.0 & 1.2 & 1.7 & 1.4 & $0.1 ; 2.7$ & $.031^{*}$ \\
\hline Point ANS SI (mm) & 0.97 & $0.90 ; 0.99$ & -1.6 & 2.6 & -0.8 & 1.3 & -0.8 & $-2.2 ; 0.6$ & .264 \\
\hline Point ANS 3D (mm) & 0.96 & $0.84 ; 0.99$ & 4.2 & 2.3 & 2.3 & 1.4 & 2.0 & $0.6 ; 3.3$ & $.005^{* *}$ \\
\hline ANS-PNS angle $\left({ }^{\circ}\right)$ & 0.95 & $0.82 ; 0.98$ & -0.6 & 2.2 & -0.1 & 1.7 & -0.5 & $-1.8 ; 0.9$ & .501 \\
\hline U1 SI (mm) & 0.96 & $0.86 ; 0.99$ & -0.3 & 2.1 & -0.2 & 1.9 & 0.0 & $-1.4 ; 1.3$ & .965 \\
\hline U1 3D (mm) & 0.91 & $0.64 ; 0.97$ & 3.4 & 1.9 & 2.9 & 2.1 & 0.6 & $-0.8 ; 2.0$ & .388 \\
\hline U6 AP (mm) & 0.99 & $0.98 ; 0.99$ & 1.4 & 2.1 & -0.6 & 1.7 & 2.0 & $0.7 ; 3.3$ & $.004^{* *}$ \\
\hline U6 SI (mm) & 0.96 & $0.84 ; 0.99$ & -1.1 & 1.1 & 0.2 & 3.4 & -1.3 & $-3.0 ; 0.4$ & .139 \\
\hline U6 3D (mm) & 0.99 & $0.96 ; 0.99$ & 3.8 & 1.3 & 3.0 & 1.0 & 0.8 & $0.0 ; 1.6$ & $.041^{*}$ \\
\hline U1 inclination $\left({ }^{\circ}\right)$ & 0.97 & $0.89 ; 0.99$ & 0.3 & 8.7 & 0.8 & 9.7 & -0.3 & $-6.6 ; 5.9$ & .914 \\
\hline U6 angulation $\left({ }^{\circ}\right)$ & 0.99 & $0.98 ; 0.99$ & 3.7 & 8.5 & -2.6 & 5.8 & 2.4 & $1.4 ; 11.3$ & $.014^{*}$ \\
\hline \multicolumn{10}{|l|}{ Mandibular skeletal changes } \\
\hline Point Pog AP (mm) & 0.99 & $0.99 ; 1.00$ & 3.3 & 2.9 & 1.8 & 2.0 & 1.6 & $-0.1 ; 3.2$ & .071 \\
\hline L1 SI (mm) & 0.94 & $0.75 ; 0.98$ & 0.4 & 2.3 & 0.2 & 1.6 & 0.1 & $-1.3 ; 2.4$ & .904 \\
\hline L1 3D (mm) & 0.90 & $0.64 ; 0.97$ & 3.6 & 2.2 & 2.8 & 1.6 & 0.6 & $-0.4 ; 2.2$ & .178 \\
\hline L6 AP (mm) & 0.99 & $0.97 ; 0.99$ & 3.1 & 1.6 & 1.4 & 1.1 & 1.9 & $0.9 ; 2.9$ & $<.001^{* * *}$ \\
\hline L6 SI (mm) & 0.97 & $0.90 ; 0.99$ & 2.0 & 1.5 & 1.3 & 1.4 & 0.6 & $-0.3 ; 1.6$ & .191 \\
\hline L6 3D (mm) & 0.92 & $0.67 ; 0.98$ & 4.7 & 1.3 & 2.8 & 1.0 & 1.9 & $1.1 ; 2.8$ & $<.001^{* * *}$ \\
\hline L1 inclination $\left({ }^{\circ}\right)$ & 0.96 & $0.51 ; 0.99$ & 5.4 & 6.1 & 5.2 & 5.2 & 0.6 & $-3.4 ; 4.6$ & .755 \\
\hline L6 angulation $\left({ }^{\circ}\right)$ & 0.96 & $0.85 ; 0.99$ & 0.4 & 5.2 & -0.6 & 3.6 & 1.4 & $-1.8 ; 4.6$ & .372 \\
\hline
\end{tabular}

Note: Positive values (anterior, superior, counterclockwise, mesial angulation and buccal inclination displacement changes. Negative values (posterior, inferior, clockwise, distal angulation and lingual inclination changes).

Abbreviations: 3D, three-dimensional; AP, anteroposterior; SI, superoinferior.

${ }^{*} P<.05$.

${ }^{* *} P<.01$.

${ }^{* * *} P<.001$.

The upper first molar presented significantly different anteroposterior, vector and angulation changes between the two groups. The Herbst group showed upper first molar changes in anterior $(1.4 \pm 2.1 \mathrm{~mm})$, inferior $(-1.1 \pm 1.1 \mathrm{~mm})$ and vector $(3.8 \pm 1.3 \mathrm{~mm})$ directions, and also mesial angulation $\left(3.7 \pm 8.5^{\circ}\right)$. The Pendulum group presented upper molar changes in posterior $(-0.6 \pm 1.7 \mathrm{~mm})$, superior $(0.2 \pm 3.4 \mathrm{~mm})$ and $3 \mathrm{D}(3.0 \pm 1.0 \mathrm{~mm})$ directions, and also distal angulation $\left(2.6^{\circ} \pm 5.8^{\circ}\right)$.

The lower incisors were protruded and proclined, without significant differences between the groups. The lower incisors displaced 
in an anterior direction in the Herbst $(2.2 \pm 2.5 \mathrm{~mm})$ and Pendulum groups $(1.7 \pm 1.9 \mathrm{~mm})$. The inclination in buccal direction also revealed similar averages in the Herbst $\left(5.4^{\circ} \pm 6.1^{\circ}\right)$ and Pendulum groups $\left(5.2^{\circ} \pm 5.2^{\circ}\right)$. The lower first molars presented significantly greater mesial movement in the Herbst group $(3.1 \pm 1.6 \mathrm{~mm}$ ) than in the Pendulum group $(1.4 \pm 1.1 \mathrm{~mm})$.

\section{4 | DISCUSSION}

This clinical investigation of pre- and post-treatment CBCT images compared two different therapeutic approaches that incorporated either a Herbst or Pendulum appliance, both followed by comprehensive fixed appliance treatment of Class II malocclusion. The analysis of starting forms revealed that the two groups were comparable at $\mathrm{T} 1$. The CVM classification revealed a similar stage of development in both groups, even with higher presence of females in the Pendulum sample. The results of this study show that there are differences between these approaches, highlighted by greater mandibular growth in the Herbst group.

\section{1 | Skeletal Changes}

A "headgear effect" on the maxilla and increased mandibular growth increments are desirable outcomes for Class II treatment. The mandibular sagittal changes in the Pendulum appliance resulted in a gain of $3.4 \mathrm{~mm}$ in growth of the mandible compared to the maxilla (difference between mandibular length and maxilla length), while in the Herbst group the same parameter showed a gain of $4.7 \mathrm{~mm}$.

The amount of anterior maxillary growth observed in the Pendulum group was significantly smaller than in the Herbst group. The small average difference between the two groups of $1.4 \mathrm{~mm}$ lesser anterior maxillary growth, measured at ANS, may indicate that the Pendulum effects on molar position and subsequent retraction of the upper teeth affected the overall maxillary growth. Previous two-dimensional studies have shown a "headgear effect" with the Herbst appliance due to the posterior and upward force vector imparted to the maxilla. ${ }^{20,21}$ In the present study, this effect was not observed. The displacement of the maxilla in the Herbst group, measured at ANS, showed $2.6 \pm 2.0 \mathrm{~mm}$ and $-1.6 \pm 2.6 \mathrm{~mm}$ in anterior and inferior directions, respectively. A similar result was observed at point $A$, presenting $1.9 \pm 1.8 \mathrm{~mm}$ and $-2.7 \pm 2.7 \mathrm{~mm}$ anterior and inferior direction, respectively.

Interestingly, the inferior displacement of Pogonion, as well as the increase in length of the mandible, was significantly greater in the Herbst group, ( $-2.2 \mathrm{~mm}$ and $2.9 \mathrm{~mm}$, respectively) than in the Pendulum group. The $2 \mathrm{~mm}$ difference in pogonion displacement between the two groups could indicate greater mandibular growth in the Herbst group. This $2 \mathrm{~mm}$ is in agreement with a previous study that reported $2.2 \mathrm{~mm}$ of $3 \mathrm{D}$ pogonion displacement in Class II patients treated without functional appliances. ${ }^{12}$ Vertical mandibular skeletal changes, as measured by alterations in the inclination of the mandibular plane, were $<1^{\circ}$, with no significant differences between the Herbst and Pendulum groups at the end of comprehensive orthodontic treatment. The amount of vertical mandibular growth, even though no rotation of the mandibular plane was observed, reduces the anteroposterior mandibular skeletal effects in both groups, as also observed by Burkhardt et al ${ }^{14}$ The average increase in anterior Pogonion projection for the Herbst group was $3.3 \mathrm{~mm} \pm 2.9 \mathrm{~mm}, 1.6 \mathrm{~mm}$ greater than in the Pendulum group, a difference that was not statistically significant.

\section{2 | Dentoalveolar changes}

In this study, a small upward and distal dentoalveolar maxillary effect at the upper first molar $(-0.6 \pm 1.7 \mathrm{~mm}$ and $0.2 \pm 3.4 \mathrm{~mm}$, respectively) was observed in the group treated with Pendulum followed by fixed appliances. Care should be taken, however, when interpreting this finding due to the small average displacement, large standard deviation and the Phase II treatment using full fixed appliances. In fact, at the end of comprehensive treatment in the group treated with Pendulum, the upper molars did not distalize bodily nor tipped distally but remained fairly upright, corroborating the findings of other studies on patients treated with Pendulum after comprehensive treatment ${ }^{5,14}$ No posterior and upward effects of the maxillary first molar following treatment with the Herbst appliance reported in other studies were seen in the current study. ${ }^{4,5,22}$

These contradictory results can be attributed to differences in the methodology (3D vs $2 \mathrm{D}$ using cephalometric variables) to assess maxillary dental positional changes. Another possible reason is that the headgear effect may have been present following Phase I part of the treatment but was diminished or negated following Phase II treatment with comprehensive fixed appliances.

Interestingly, the lower molar presented significantly greater mesial displacement in the Herbst group than in the Pendulum group (3.1 $\pm 1.6 \mathrm{~mm}$ and $1.4 \pm 1.1 \mathrm{~mm}$, respectively), probably due to differences in the forces applied by the Herbst appliance on the lower teeth compared to the Pendulum appliance.

The small maxillary incisor (U1) proclination and anteroposterior displacement were not significantly different between the two groups. The Pendulum and Herbst groups showed proclination changes of $0.3^{\circ} \pm 8.7^{\circ}$ and $0.8^{\circ} \pm 9.7^{\circ}$, and anteroposterior displacement of $0.1 \mathrm{~mm} \pm 2.6 \mathrm{~mm}$ and $0.5 \pm 2.7 \mathrm{~mm}$, respectively. The $2 \mathrm{D}$ cephalometry literature also reports similar ranges of variability of upper incisor proclination in patients treated with the Herbst appliance and Pendulum appliances. . $14,20,23,24$ The small average anterior displacement and proclination of the upper incisors in the Herbst group in the current 3D study differs from previous investigations that reported posterior displacement of the upper incisors between $0.2 \mathrm{~mm}^{23}$ and $2.8 \mathrm{~mm}^{25}$ The lack of between-group differences in incisor proclination in the present study could be attributed to the large variability among patients in this study and to Phase II treatment with full fixed appliances.

Proclination and protrusion of the lower incisors are attributed more frequently to mandibular advancement appliances, ${ }^{26}$ such as the Herbst treatment and less to distalization methods, such as 
FIGURE 2 Summary of the skeletal and dentoalveolar effects of Class II treatment using Herbst and fixed appliance. A-Cranial base superimposition and maxillomandibular changes; B-Maxillary superimposition and dentoalveolar changes in upper molar and incisors; C-Mandibular superimposition and lower molar and incisor changes [Colour figure can be viewed at wileyonlinelibrary.com]
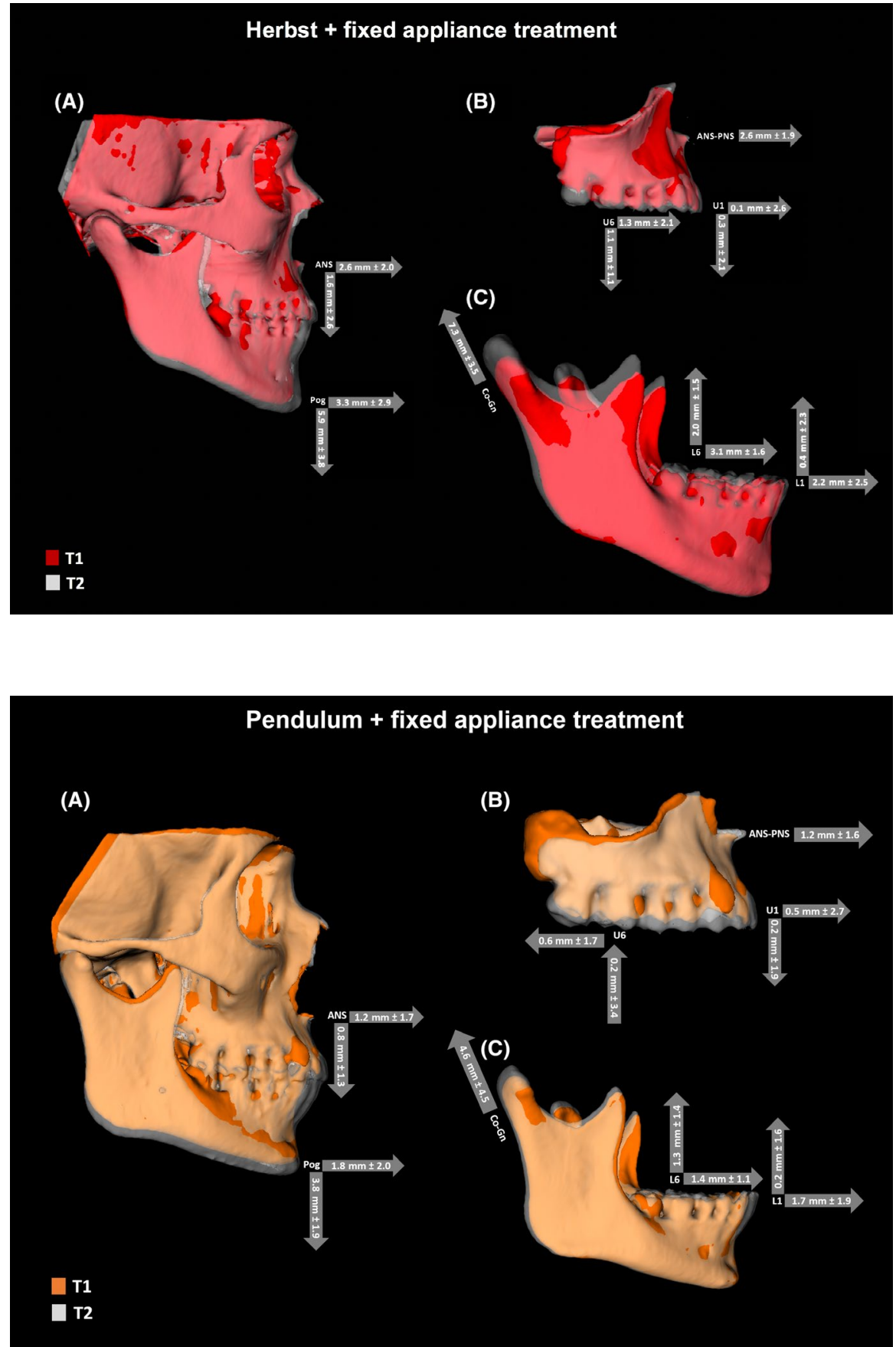

FIGURE 3 Summary of the skeletal and dentoalveolar effects of Class II treatment using Pendulum and fixed appliance. A-Cranial base superimposition and maxillomandibular changes; B-Maxillary superimposition and dentoalveolar changes in upper molar and incisors; C- Mandibular superimposition and lower molar and incisor changes [Colour figure can be viewed at wileyonlinelibrary.com] with the Pendulum appliance. Our findings showed no significant between-group differences, with average difference of $0.5 \mathrm{~mm}$ and $0.6^{\circ}$ in lower incisor protrusion and proclination, respectively. These results are in agreement with those of Burkhardt et al ${ }^{14}$

\section{3 | Overall treatment effects}

This study evaluated changes after two phases of treatment, Phase 1 Class II correction (Herbst or Pendulum appliances) and Phase II detailing of the occlusion with fixed appliances. The combination of skeletal and dentoalveolar effects at the end of treatment led to a similar overall correction of the maxillomandibular discrepancy, although each approach had different influences in the maxilla or mandible (Figures 2 and 3). Herbst treatment followed by fixed appliances corrected the Class II malocclusion, with greater effects in the lower arch, leading to a differential maxillomandibular growth of $4.7 \mathrm{~mm}$ mandibular gain (CoGn-ANS-PNS), and $2.3 \mathrm{~mm}$ greater mesial displacement of the lower first molar than the upper first molar (U6-L6 AP).

This study is limited in that specific treatment effect of either type of Class II corrector cannot be determined with precision because a CBCT image was not taken at the end of Phase I for ethical reasons and ALARA principle, balancing the risks and benefits, 
especially in young populations. ${ }^{27}$ Another limitation of the present study is its retrospective design, as a prospective study would allow better control of the treatment protocols and monitoring of the clinical variables. The sample size calculation was performed as described, while $2 \mathrm{~mm}$ for statistical significance may be questionable and controversial clinically. Future studies could assess how much, in $\mathrm{mm}$, can prove to be clinically significant and also bring additional information about the skeletal and dentoalveolar effects of the Pendulum and Herbst treatment with orthodontic fixed appliances.

The results of this study provided insights for clinical choice for Class II correction. Dentoalveolar effects combined with skeletal changes contributed to the correction of the Class II malocclusion, with opposite effects on the upper posterior teeth in the Herbst and pendulum groups and similar dentoalveolar effects on the anterior teeth. The 3D changes, and their anteroposterior and superoinferior components, showed that these Class II treatment approaches in this study both achieve Class II correction by different mechanisms. The large patient variability in response to treatment in both the Herbst and Pendulum groups, however, revealed that within each group there were patients who responded well, and some who did not, to the predicted molar distalization in Pendulum group or anterior mandibular positioning in the Herbst group. Given the study results, the decision between different types of therapies in non-surgical Class II patients can focus on the magnitude of skeletal and dentoalveolar needs and also on the priority regions of desired changes.

\section{5 | CONCLUSIONS}

- The differential maxillomandibular growth contributed to the correction of the Class II malocclusion in patients treated either with Herbst or with Pendulum followed up by fixed appliances with overall downward and forward maxillary and mandibular skeleta displacement relative to the cranial base.

- The growth response was significantly greater in the Herbst group for maxillary and mandibular length.

- The directional changes showed greater inferior displacement of Pogonion and anterior displacement of maxilla (ANS) in the Herbst group.

- The upper first molar in the Pendulum group showed slight distal displacement in the opposite direction compared to the Herbst group.

- The lower first molar showed greater mesial movement in the Herbst group.

- Both treatment groups showed similar amount of proclination and protrusion of the lower incisors.

\section{ACKNOWLEDGEMENTS}

This study was financed by NIH Grant NIDCR R01 DE024450 and in part by the Coordenação de Aperfeiçoamento de Pessoal de Nível Superior-Brasil (CAPES)-Finance Code 001.

\section{CONFLICT OF INTEREST}

All authors have nothing to disclose.

\section{ORCID}

Kyle L. Taylor iD https://orcid.org/0000-0002-9256-8570

Karine Evangelista iD https://orcid.org/0000-0003-0258-9739

Luciana Muniz (D) https://orcid.org/0000-0001-6832-080X

Antônio Carlos de Oliveira Ruellas (iD https://orcid. org/0000-0001-7973-9286

José Valladares-Neto (iD https://orcid.org/0000-0003-0147-1931

Lorenzo Franchi (iD https://orcid.org/0000-0002-2072-460X

Hera Kim-Berman (iD https://orcid.org/0000-0003-4465-3700

Lucia Helena Soares Cevidanes (iD https://orcid.

org/0000-0001-9786-2253

\section{REFERENCES}

1. Pancherz $\mathrm{H}$. Treatment of class II malocclusions by jumping the bite with the Herbst appliance. A cephalometric investigation. Am J Orthod. 1979;76:423-442.

2. Bussick TJ, McNamara JA Jr. Dentoalveolar and skeletal changes associated with the pendulum appliance. Am J Orthod Dentofac Orthop. 2000;117:333-343.

3. Hilgers JJ. The pendulum appliance for class II non-compliance therapy. J Clin Orthod. 1992;26:706-714.

4. Nelson B, Hansen K, Hägg U. Class II correction in patients treated with class II elastics and with fixed functional appliances: a comparative study. Am J Orthod Dentofacial Orthop. 2000;118:142-149.

5. Tomblyn T, Rogers M, Andrews L, et al. Cephalometric study of Class II division 1 patients treated with an extended-duration, reinforced, banded Herbst appliance followed by fixed appliances. Am J Orthod Dentofacial Orthop. 2016;150:818-830.

6. Caprioglio A, Fontana M, Longoni E, Cozzani M. Long-term evaluation of the molar movements following Pendulum and fixed appliances. Angle Orthod. 2013;83:447-454.

7. Fuziy A, de Almeida RR, Janson G, Angelieri F, Pinzan A. Sagittal, vertical, and transverse changes consequent to maxillary molar distalization with the pendulum appliance. Am J Orthod Dentofac Orthop. 2006;130:502-510.

8. Cozza P, Baccetti T, Franchi L, De Toffol L, McNamara JA. Mandibular changes produced by functional appliances in class II malocclusion: a systematic review. Am J Orthod Dentofacial Orthop. 2006;129(5):599.e1-599.e12.

9. Rogers K, Campbell PM, Tadlock L, Schneiderman E, Buschang PH. Treatment changes of hypo- and hyperdivergent class II Herbst patients. Angle Orthod. 2018;88:3-9.

10. Rabie AB, Zhao Z, Shen G, Hägg EU, Dr O, Robinson W. Osteogenesis in the glenoid fossa in response to mandibular advancement. Am J Orthod Dentofacial Orthop. 2001;119:390-400.

11. Baccetti T, Franchi L, Stahl F. Comparison of 2 comprehensive Class II treatment protocols including the bonded Herbst and headgear appliances: a double-blind study of consecutively treated patients at puberty. Am J Orthod Dentofacial Orthop. 2009;135(6):698. e1-698.e10.

12. Souki BQ, Vilefort P, Oliveira DD, et al. Three-dimensional skeletal mandibular changes associated with Herbst appliance treatment. Orthod Craniofac Res. 2017;20:111-118. 
13. Atresh A, Cevidanes L, Yatabe M, et al. Three-dimensional treatment outcomes in class II patients with different vertical facial patterns treated with the Herbst appliance. Am J Orthod Dentofacial Orthop. 2018;154(2):238-248.e1.

14. Burkhardt DR, McNamara JA Jr, Baccetti T. Maxillary molar distalization or mandibular enhancement: a cephalometric comparison of comprehensive orthodontic treatment including the pendulum and the Herbst appliances. Am J Orthod Dentofac Orthop. 2003;123:108-116.

15. McNamara JA Jr, Franchi L. The cervical vertebral maturation method: a user's guide. Angle Orthod. 2018;88:133-143.

16. Ruellas A, Tonello C, Gomes LR, et al. Common 3-dimensional coordinate system for assessment of directional changes. Am J Orthod Dentofac Orthop. 2016;149:645-656.

17. Cevidanes L, Styner MA, Proffit WR. Image analysis and superimposition of 3-dimensional cone-beam computed tomography models. Am J Orthod Dentofac Orthop. 2006;129:611-618.

18. Ruellas ACdO, Huanca Ghislanzoni LT, Gomes MR, et al. Comparison and reproducibility of 2 regions of reference for maxillary regional registration with cone-beam computed tomography. Am J Orthod Dentofacial Orthop. 2016;149:533-542.

19. De Oliveira Ruellas AC, Yatabe MS, Souki BQ, et al. 3D mandibular superimposition: comparison of regions of reference for voxelbased registration. PLoS ONE. 2016;11:1-13.

20. Pancherz H, Anehus-Pancherz M. The headgear effect of the Herbst appliance: a cephalometric long-term study. Am J Orthod Dentofacial Orthop. 1993;103:510-520.

21. Wigal TG, Dischinger T, Martin C, Razmus T, Gunel E, Ngan P. Stability of class II treatment with an edgewise crowned Herbst appliance in the early mixed dentition: Skeletal and dental changes. Am J Orthod Dentofac Orthop. 2011;140:210-223.
22. Barnett GA, Higgins DW, Major PW, Flores-Mir C. Immediate skeletal and dentoalveolar effects of the crown- or banded type Herbst appliance on class II division 1 malocclusion. Angle Orthod. 2008;78:361-369.

23. Lai M, McNamara JA Jr. An evaluation of two-phase treatment with the Herbst appliance and preadjusted edgewise therapy. Semin Orthod. 1998;4:46-58.

24. Chiu PP, McNamara JA Jr, Franchi L. A comparison of two intraoral molar distalization appliances: distal jet versus pendulum. Am J Orthod Dentofac Orthop. 2005;128:353-365.

25. Hansen K, Pancherz H, Hägg U. Long-term effects of the Herbst appliance in relation to the treatment growth period: a cephalometric study. Eur J Orthod. 1991;13:471-481.

26. Martin J, Pancherz H. Mandibular incisor position in relation to amount of bite jumping during Herbst/multibracket appliance treatment: a radiographic-cephalometric study. Am J Orthod Dentofac Orthop. 2009;136:44-51.

27. Oenning AC, Jacobs R, Pauwels R, Straits A, Hedesiu M, Salmon B. Cone-beam CT in paediatric dentistry: DIMITRA project position statement. Pediatr Radiol. 2018;48:308-316.

How to cite this article: Taylor KL, Evangelista K, Muniz L, et al. Three-dimensional comparison of the skeletal and dentoalveolar effects of the Herbst and Pendulum appliances followed by fixed appliances: A CBCT study. Orthod Craniofac Res. 2020;23:72-81. https://doi.org/10.1111/ocr.12345 